\title{
ON THE LIMIT OF THE COEFFICIENTS OF THE EIGEN- FUNCTION SERIES ASSOCIATED WITH A CERTAIN NON-SELF-ADJOINT DIFFERENTIAL SYSTEM ${ }^{1}$
}

\author{
LUNA I. MISHOE AND GLORIA C. FORD
}

Introduction. In the attempt to solve certain problems in mathematical-physics, such as diffraction of an arbitrary pulse by a wedge as considered by Irvin Kay [1], one encounters the hyperbolic differential equation

$$
u_{x x}-q(x) u=u_{x t}-p(x) u_{t}
$$

where $u(x, t)$ must satisfy the conditions $u(a, t)=u(b, t)=0$ and $u(x, 0)=F(x)$. In attempting to solve equation (1) by separation of variables, one is led to the consideration of expanding an arbitrary function $F(x)$ in terms of the eigenfunctions, or nonzero solutions, $u_{n}(x)$ of the equation:

$$
(A+\lambda B) u=0
$$

satisfying the conditions $u(a)=u(b)=0$, where $A$ is the operator $d^{2} / d x^{2}+q(x)$ and $B$ is the operator $-d / d x+p(x)$. The system adjoint to $(2)$ is:

$$
\left(A^{*}+\lambda B^{*}\right) v=0, \quad v(a)=v(b)=0
$$

where $A=A^{*}$ and $B^{*}=d / d x+p(x)$.

Conditions have been established [2], under which a function $F(x)$ of bounded variation on $(a, b)$ can be expanded in terms of $u_{n}(x)$. However, in the expansion $F(x)=\sum_{-\infty}^{\infty} a_{n} u_{n}(x)$ there are certain properties of the coefficients, $a_{n}$, which differ quite radically from the corresponding properties of the coefficients of certain well-known selfadjoint eigenfunction expansions. For example, if $B_{n}$ are the Fourier coefficients of a function $g(x)$, it is well known that $\lim _{n \rightarrow \infty} B_{n}=0$. However, in the expansion $F(x)=\sum_{-\infty}^{\infty} a_{n} u_{n}(x)$, it is found that $\lim _{n \rightarrow \infty} a_{n}$ is not in general equal to zero. Consequently, the series $\sum_{1}^{\infty} a_{n}^{2}$, unlike the corresponding series of Fourier coefficients, does not in general converge.

Received by the editors August 17, 1953 and, in revised form, May 4, 1955.

1 The research in this document has been made possible through a grant to Morgan State College by the National Science Foundation, Washington, D. C. Research Grant NSF-G 1224, November 1954. 
In this paper it is proved that $\lim _{n \rightarrow \infty} a_{n}=0$ if and only if $F(a)$ $=F(b)=0$.

The following theorem was proved in [2]:

THEOREM 1. Let $q(x)$ be continuous and let $p(x)$ have a continuous second derivative. If $F(x)$ is of bounded variation in $(a, b)$ and if

$$
F(a+0)+F(b-0) \exp \left[-\int_{a}^{b} p(t) d t\right]=0,
$$

then the series

$$
\sum_{-\infty}^{\infty} a_{n} u_{n}(x)
$$

where

$$
a_{n}=\frac{\int_{a}^{b} F(\xi) B^{*} v_{n}(\xi) d \xi}{\int_{a}^{b} u_{n}(\xi) B^{*} v_{n}(\xi) d \xi}
$$

with $u_{n}(x)$ and $v_{n}(x)$ eigenfunctions of (2) and (3) respectively, converges to $[F(x+0)+F(x-0)] / 2$ in the interval $a<x<b$. If $F(x)$ does not satisfy the condition (4), then the series (5) converges to

$$
T(x)=[F(x+0)+F(x-0)] / 2-c \exp \left[\int_{a}^{x} p(t) d t\right]
$$

in the interval $a<x<b$, where

$$
c=\left\{F(a+0)+F(b-0) \exp \left[-\int_{a}^{b} p(t) d t\right]\right\} / 2 .
$$

We now prove:

THEOREM 2. If $F^{\prime}(x)$ exists and is of bounded variation for $a \leqq x \leqq b$, and if $F(a)+F(b) \exp \left[-\int_{a}^{b} p(t) d t\right]=0$, then a necessary and sufficient condition that $\lim _{n \rightarrow \infty} a_{n}=0$ is that $F(a)=F(b)=0$. (The prime denotes differentiation with respect to $x$.)

Asymptotic form of $a_{n}$. Since

$$
a_{n}=\int_{a}^{b} F(\xi) B^{*} v_{n}(\xi) d \xi / \int_{a}^{b} u_{n}(\xi) B^{*} v_{n}(\xi) d \xi,
$$

we can develop the asymptotic form for $a_{n}$ by considering the corresponding forms for $u_{n}(\xi)$ and $B^{*} v_{n}(\xi)$, and we have from [2] 
$u_{n}(x)=u_{a}\left(x, \lambda_{n}\right)$

(6)

$$
\begin{aligned}
= & \lambda_{n}^{-1}\left\{\exp \left[\lambda_{n}(x-a)-\int_{a}^{x} p(t) d t\right]-\exp \left[\int_{a}^{x} p(t) d t\right]\right\} \\
+O\left(\lambda_{n}^{-2} \exp \left[\left(\lambda_{n}+|\sigma|\right)(x-a) / 2\right]\right), & \text { where } \sigma_{n}=\operatorname{Re} \lambda_{n} \text { and }\left|\lambda_{n}\right| \rightarrow \infty,
\end{aligned}
$$

(7) $B^{*} v_{n}(x)=B^{*} v_{a}\left(x, \lambda_{n}\right)=\exp \left[-\lambda_{n} x+\int_{a}^{x} p(t) d t\right]+\Omega_{a}$,

where

$$
\Omega_{a}= \begin{cases}O\left(\lambda_{n}^{-2} \exp \left[-\lambda_{n} a\right]\right)+O\left(\lambda_{n}^{-1} \exp \left[-\lambda_{n} x\right]\right), & \operatorname{Re} \lambda_{n} \geqq 0 \\ O\left(\lambda_{n}^{-1} \exp \left[-\lambda_{n} x\right]\right) & \operatorname{Re} \lambda_{n} \leqq 0\end{cases}
$$

and where

(7a)

$$
\lambda_{n}=\frac{2 n \pi i+2 \int_{a}^{b} p(t) d t+o\left(\frac{1}{n}\right)}{b-a} .
$$

From (6) and (7) we have:

$$
\begin{aligned}
u_{n}(\xi) B^{*} v_{n}(\xi)= & \frac{\exp \left(-\lambda_{n} a\right)}{\lambda_{n}}+o\left(\frac{1}{\lambda_{n}^{2}}\right) \\
& -\frac{\exp \left[-\lambda_{n} \xi+2 \int_{a}^{\xi} p(t) d t\right]}{\lambda_{n}}
\end{aligned}
$$

and

$$
\int_{a}^{b} u_{n}(\xi) B^{*} v_{n}(\xi) d \xi=\int_{a}^{b} \frac{\exp \left(-\lambda_{n} a\right)}{\lambda_{n}} d \xi+\int_{a}^{b} O\left(\frac{1}{\lambda_{n}^{2}}\right) d \xi
$$

$$
-\int_{a}^{b} \frac{\exp \left[-\lambda_{n} \xi+2 \int_{a}^{\xi} p(t) d t\right]}{\lambda_{n}} d \xi
$$

Now since $u_{n}(\xi)$ has a bounded derivative on $(a, b)$, it follows that $u_{n}(\xi)$ is of bounded variation for $a \leqq \xi \leqq b$. Also

$$
B^{*} v_{n}(\xi)=p(\xi) v_{n}(\xi)+v_{n}^{\prime}(\xi)
$$

and 


$$
\frac{d}{d \xi}\left[B^{*} v_{n}(\xi)\right]=p(\xi) v_{n}^{\prime}(\xi)+v_{n}(\xi) p^{\prime}(\xi)+v_{n}^{\prime \prime}(\xi)
$$

but by (3)

$$
v_{n}^{\prime \prime}(\xi)=-q(\xi) v_{n}(\xi)-\lambda_{n}\left[p(\xi) v_{n}(\xi)+v_{n}^{\prime}(\xi)\right] .
$$

Therefore

$$
\begin{aligned}
\frac{d}{d \xi}\left[B^{*} v_{n}(\xi)\right]= & p(\xi) v_{n}^{\prime}(\xi)+v_{n}(\xi) p^{\prime}(\xi)-q(\xi) v_{n}(\xi) \\
& -\lambda_{n}\left[p(\xi) v_{n}(\xi)+v_{n}^{\prime}(\xi)\right]
\end{aligned}
$$

is bounded for $a \leqq \xi \leqq b$. Hence $B^{*} v_{n}(\xi)$ is of bounded variation for $a \leqq \xi \leqq b$ and it follows that

$$
u_{n}(\xi) B^{*} v_{n}(\xi)
$$

is of bounded variation, and consequently the term

$$
O\left(\frac{1}{\lambda_{n}^{2}}\right) \equiv \frac{g(n, \xi)}{\lambda_{n}^{2}}
$$

in (9) is of bounded variation for $a \leqq \xi \leqq b$. Now put

$$
g(n, \xi)=Q_{1}(n, \xi)-Q_{2}(n, \xi)
$$

where $Q_{1}(n, \xi)$ and $Q_{2}(n, \xi)$ are two non-negative bounded monotone decreasing functions, and apply the mean value theorem to (9) and we get:

$$
\begin{aligned}
\int_{a}^{b} u_{n}(\xi) B^{*} \eta_{n}(\xi) d \xi & \\
= & \int_{a}^{b} \frac{\exp \left(-\lambda_{n} a\right)}{\lambda_{n}} d \xi+\frac{Q_{1}(n, a)}{\lambda_{n}^{2}} \int_{a}^{d_{1}} d \xi \\
& -\frac{Q_{2}(n, a)}{\lambda_{n}^{2}} \int_{a}^{d_{2}} d \xi-\int_{a}^{b} \frac{\exp \left[-\lambda_{n} \xi+2 \int_{a}^{\xi} p(t) d t\right]}{\lambda_{n}} d \xi \\
= & \frac{(b-a) \exp \left(-\lambda_{n} a\right)}{\lambda_{n}}+O\left(\frac{1}{\lambda_{n}^{2}}\right) \\
= & \frac{\left.(b-a) \exp \left(-\lambda_{n} a\right)+O\left(\frac{1}{\lambda_{n}}\right)\right]}{\lambda_{n}}, \text { where } a<d_{1}, d_{2}<b .
\end{aligned}
$$


Now put

$$
\left[(b-a) \exp \left(-\lambda_{n} a\right)+O\left(\frac{1}{\lambda_{n}}\right)\right]=\frac{1}{B(n)} .
$$

We then have

$$
\begin{aligned}
a_{n}= & B(n) \lambda_{n} \int_{a}^{b} F(\xi) \exp \left[-\lambda_{n} \xi+\int_{a}^{\xi} p(t) d t\right] d \xi \\
& +B(n) \lambda_{n} \int_{a}^{b} \frac{F(\xi) O\left[\exp \left(-\lambda_{n} \xi\right)\right]}{\lambda_{n}} d \xi \\
& +B(n) \lambda_{n} \int_{a}^{b} F(\xi) O\left(\frac{\exp \left[-\lambda_{n} a\right]}{\lambda_{n}^{2}}\right) d \xi, \quad \text { for } \operatorname{Re} \lambda_{n} \geqq 0 \\
a_{n}= & B(n) \lambda_{n} \int_{a}^{b} F(\xi) \exp \left[-\lambda_{n} \xi+\int_{a}^{\xi} p(t) d t\right] d \xi \\
& +B(n) \lambda_{n} \int_{a}^{b} F(\xi) O\left(\frac{\exp \left[-\lambda_{n} \xi\right]}{\lambda_{n}}\right) d \xi, \quad \operatorname{Re} \lambda_{n} \leqq 0 .
\end{aligned}
$$

Determination of the limit of $a_{n}$. From equation (14) we have, for $\operatorname{Re} \lambda_{n} \geqq 0$ :

$$
\begin{aligned}
\lim _{n \rightarrow \infty} a_{n}= & \lim _{n \rightarrow \infty} B(n) \lambda_{n} \int_{a}^{b} F(\xi) \exp \left[-\lambda_{n} \xi+\int_{a}^{\xi} p(t) d t\right] d \xi \\
& +\lim _{n \rightarrow \infty} B(n) \int_{a}^{b} F(\xi) O\left(\exp \left[-\lambda_{n} \xi\right]\right) d \xi \\
& +\lim _{n \rightarrow \infty} \frac{B(n)}{\lambda_{n}} \int_{a}^{b} F(\xi) O\left(\exp \left[-\lambda_{n} a\right]\right) d \xi,
\end{aligned}
$$

provided these limits exist. Since $B *_{n}(x)$ is of bounded variation for $a \leqq x \leqq b$, it is clear from (7) that the expressions $O\left(\exp \left[-\lambda_{n} \xi\right]\right)$ and $O\left(\exp \left[-\lambda_{n} a\right]\right)$ in the in tegrands of (16) are also of bounded variation for $a \leqq \xi \leqq b$.

Consider now the second integral of (16). We have:

$$
\begin{aligned}
\lim _{n \rightarrow \infty} B(n) \int_{a}^{b} F(\xi) O(\exp [ & \left.\left.-\lambda_{n} \xi\right]\right) d \xi \\
& =\lim _{n \rightarrow \infty} B(n) \int_{a}^{b} F(\xi) g_{1}(n, \xi) \exp \left[-\lambda_{n} \xi\right] d \xi,
\end{aligned}
$$

where $g_{1}(n, \xi)$ and consequently $F(\xi) g_{1}(n, \xi)$ are of bounded variation 
for $a \leqq \xi \leqq b$. Put $F(\xi) g_{1}(n, \xi)=Q_{3}(n, \xi)-Q_{4}(n, \xi)$, where $Q_{3}(n, \xi)$ and $Q_{4}(n, \xi)$ are two non-negative, bounded monotone decreasing functions, and apply the mean value theorem to (17) and we get:

$$
\begin{aligned}
& \lim _{n \rightarrow \infty} B(n) \int_{a}^{b} F(\xi) g_{1}(n, \xi) \exp \left[-\lambda_{n} \xi\right] d \xi \\
& \left.\left.(18) \quad=\lim _{n \rightarrow \infty} B(n)\left[\frac{Q_{3}(n, a) \exp \left[-\lambda_{n} \xi\right]}{-\lambda_{n}}\right)_{a}^{d_{3}}+\frac{Q_{4}(n, a) \exp \left[-\lambda_{n} \xi\right]}{\lambda_{n}}\right)_{a}^{d_{4}}\right] \\
& =\lim _{n \rightarrow \infty} \frac{O(1)}{\lambda_{n}} .
\end{aligned}
$$

Since $\lambda_{n}=\left(2 n \pi i+2 \int_{a}^{b} p(t) d t\right) /(b-a)+O(1 / n)$, it is clear that $\lambda_{n}=n O(1)$ and (18) becomes:

$$
\lim _{n \rightarrow \infty} B(n) \int_{a}^{b} F(\xi) g_{1}(n, \xi) \exp \left[-\lambda_{n} \xi\right] d \xi=\lim _{n \rightarrow \infty} \frac{O(1)}{n}=0 .
$$

A completely similar argument will show that the third integral in (16) becomes:

$$
\lim _{n \rightarrow \infty} \frac{B(n)}{\lambda_{n}} \int_{a}^{b} F(\xi) O\left(\exp \left[-\lambda_{n} a\right]\right) d \xi=\lim _{n \rightarrow \infty} \frac{O(1)}{n}=0 .
$$

Hence, by (14) and (15), we have:

$$
\lim _{n \rightarrow \infty} a_{n}=\lim _{n \rightarrow \infty} \lambda_{n} B(n) \int_{a}^{b} F(\xi) \exp \left[-\lambda_{n} \xi+\int_{a}^{\xi} p(t) d t\right] d \xi .
$$

If in (20), we put $F(\xi) \exp \left[\int_{a}^{\xi} p(t) d t\right]=H(\xi)$ and integrate by parts we get:

$$
\begin{aligned}
\lim _{n \rightarrow \infty} a_{n}= & -\lim _{n \rightarrow \infty} B(n)\left\{\left[H(\xi) \exp \left(-\lambda_{n} \xi\right)\right]_{a}^{b}\right. \\
& \left.-\int_{a}^{b} H^{\prime}(\xi) \exp \left(-\lambda_{n} \xi\right) d \xi\right\} .
\end{aligned}
$$

Using the fact that $F^{\prime}(\xi)$ is of bounded variation for $a \leqq \xi \leqq b$, it follows that $H^{\prime}(\xi)$ is also of bounded variation for $a \leqq \xi \leqq b$ and

$$
\lim _{n \rightarrow \infty} \int_{a}^{b} H^{\prime}(\xi) \exp \left(-\lambda_{n} \xi\right) d \xi=\lim _{n \rightarrow \infty} O\left(\frac{1}{\lambda_{n}}\right)=\lim _{n \rightarrow \infty} \frac{O(1)}{n}=0 .
$$

We have finally, by (7a), (21) and (22): 


$$
\begin{aligned}
\lim _{n \rightarrow \infty} a_{n}= & \lim _{n \rightarrow \infty} B(n)\left\{F(a) \exp \left[-\lambda_{n} a\right]\right. \\
& \left.-F(b) \exp \left[\int_{a}^{b} p(t) d t-\lambda_{n} b\right]\right\} .
\end{aligned}
$$

But, by Theorem 2, we have

$$
F(a)=-\exp \left[-\int_{a}^{b} p(t) d t\right] F(b) .
$$

Therefore

$$
\begin{gathered}
\lim _{n \rightarrow \infty} a_{n}=-F(b) \lim _{n \rightarrow \infty} B(n)\left(\exp \left[-\int_{a}^{b} p(t) d t-\lambda_{n} a\right]\right. \\
\left.+\exp \left[\int_{a}^{b} p(t) d t-\lambda_{n} b\right]\right) .
\end{gathered}
$$

We have from (13) that:

$$
\lim _{n \rightarrow \infty} B(n) e^{-\lambda_{n} a}=\frac{1}{b-a} .
$$

Using this result in (25), we have

$$
\begin{array}{r}
\lim _{n \rightarrow \infty} a_{n}=\frac{-F(b)}{b-a} \lim _{n \rightarrow \infty}\left(\exp \left[-\int_{a}^{b} p(t) d t-2 \lambda_{n} a\right]\right. \\
\left.+\exp \left[\int_{a}^{b} p(t) d t-\lambda_{n}(b+a)\right]\right) .
\end{array}
$$

From the value of $\lambda_{n}$ as given by (7a), it is clear that the second factor in equation (26) does not approach zero as $n$ approaches infinity. Hence it follows that $\lim _{n \rightarrow \infty} a_{n}=0$ if and only if $F(b)=0$. Then it follows from (24) that $F(a)$ is also equal to zero. And, our theorem is proved.

\section{REFERENCES}

1. Irvin Kay, Diffraction of an arbitrary pulse by a wedge, Mathematics Research Group, New York University, Report No. EM-43.

2. B. Friedman and L. I. Mishoe, Eigenfunction expansions associated with a nonself-adjoint differential equation, Institute of Mathematical Sciences, New York University, Research Report No. BR-4, 1954.

Morgan State College 